\title{
Experimental Validation: Subscale Aircraft Ground Facilities and Integrated Test Capability
}

\author{
Roger M. Bailey ${ }^{*}$, Robert W. Hostetler ${ }^{\dagger}$, Kevin N. Barnes ${ }^{\ddagger}$, \\ Celeste M. Belcastro ${ }^{\S}$, and Christine M. Belcastro ${ }^{* *}$ \\ NASA Langley Research Center, Hampton VA 23681
}

\begin{abstract}
Experimental testing is an important aspect of validating complex integrated safetycritical aircraft technologies. The Airborne Subscale Transport Aircraft Research (AirSTAR) Testbed is being developed at NASA Langley to validate technologies under conditions that cannot be flight validated with full-scale vehicles. The AirSTAR capability comprises a series of flying sub-scale models, associated ground-support equipment, and a base research station at NASA Langley. The subscale model capability utilizes a generic 5.5\% scaled transport class vehicle known as the Generic Transport Model (GTM). The AirSTAR Ground Facilities encompass the hardware and software infrastructure necessary to provide comprehensive support services for the GTM testbed. The ground facilities support remote piloting of the GTM aircraft, and include all subsystems required for data/video telemetry, experimental flight control algorithm implementation and evaluation, GTM simulation, data recording/archiving, and audio communications. The ground facilities include a self-contained, motorized vehicle serving as a mobile research command/operations center, capable of deployment to remote sites when conducting GTM flight experiments. The ground facilities also include a laboratory based at NASA LaRC providing near identical capabilities as the mobile command/operations center, as well as the capability to receive data/video/audio from, and send data/audio to the mobile command/operations center during GTM flight experiments.
\end{abstract}

\subsection{Introduction}

The Single Aircraft Accident Prevention Project of the NASA Aviation Safety and Security Program (AvSSP) is developing technologies to reduce aircraft accidents resulting from loss of vehicle control as well as failures [1]. Loss-of-control is among the highest accident categories across all vehicle classes for both the number of accidents and the number of fatalities. Although many loss-of-control accidents include system or component failures as a primary or secondary causal factor, aircraft accidents due to failures have also resulted in a significant number of fatal accidents that can be listed as a separate category. The research goal is to make revolutionary enhancements to aviation safety by improving the ability to monitor vehicle health and to safely manage fault and failure conditions in aircraft systems, and to prevent and recover from aircraft loss-of-control conditions. The scope of research encompasses all aspects of the hardware and software life cycle, from analysis of design specifications, implementation and verification, to validation methods to ensure design correctness, fault tolerance, and functional integrity in the operating environment. A validation process is currently under development that includes a coordinated approach to analysis, simulation, and experimental test methods [2] - [3]. Experimental testing includes the Airborne Sub-scale Transport Aircraft Research (AirSTAR) testbed capability that is being developed to validate technologies that cannot be flight validated with full-scale vehicles. Dynamics modeling and simulation technologies for adverse and upset flight conditions require flight validation at extreme and upset conditions, as well as unusual flight maneuvers within the normal flight envelope. Detection and identification technologies for failures and damage under adverse and upset flight conditions will require flight validation under normal, extreme, and upset flight conditions. Upset recovery algorithms, reconfigurable control for failures and damage, and adaptive outer-

\footnotetext{
* Senior Research Engineer, Safety-Critical Avionics Systems, 1 S. Wright St., Mail Stop 130.

${ }^{\dagger}$ Research Engineer, Safety-Critical Avionics Systems, 1 S. Wright St., Mail Stop 130.

${ }^{\ddagger}$ Research Engineer, Safety-Critical Avionics Systems, 1 S. Wright St., Mail Stop 130.

${ }^{\S}$ Senior Research Engineer and Technical Manager, Safety-Critical Avionics Systems, 1 S. Wright St., Mail Stop 130, AIAA Senior Member.

** Senior Research Engineer and Technical Manager, Dynamic Systems and Control, 8 Langley Blvd., Mail Stop 308, AIAA Senior Member.
} 
loop autopilot and trajectory management algorithms for damage and failure conditions will also require flight validation under extreme and upset flight conditions. These validation requirements are unsuitable for full-scale flight validation due to high risk, but can be satisfied using sub-scale models.

Use of a Remotely Piloted Vehicle for conducting flight tests to validate control upset prevention and recovery technologies (including modeling and control methods for characterizing and recovering from upset conditions) will require a robust and reliable ground support system for pilots and researchers. This paper describes the ground facilities being developed to support the NASA Langley Research Center AirSTAR testbed. The AirSTAR aircraft is a 5.5\% dynamically scaled, remotely piloted, Generic Transport Model (GTM) which will be used to conduct flight test research experiments pertaining to dynamics modeling and control beyond the normal flight envelope [4] - [5]. Figure 1 shows a functional diagram of the basic GTM-Ground Facilities (GTM-GF) architecture.

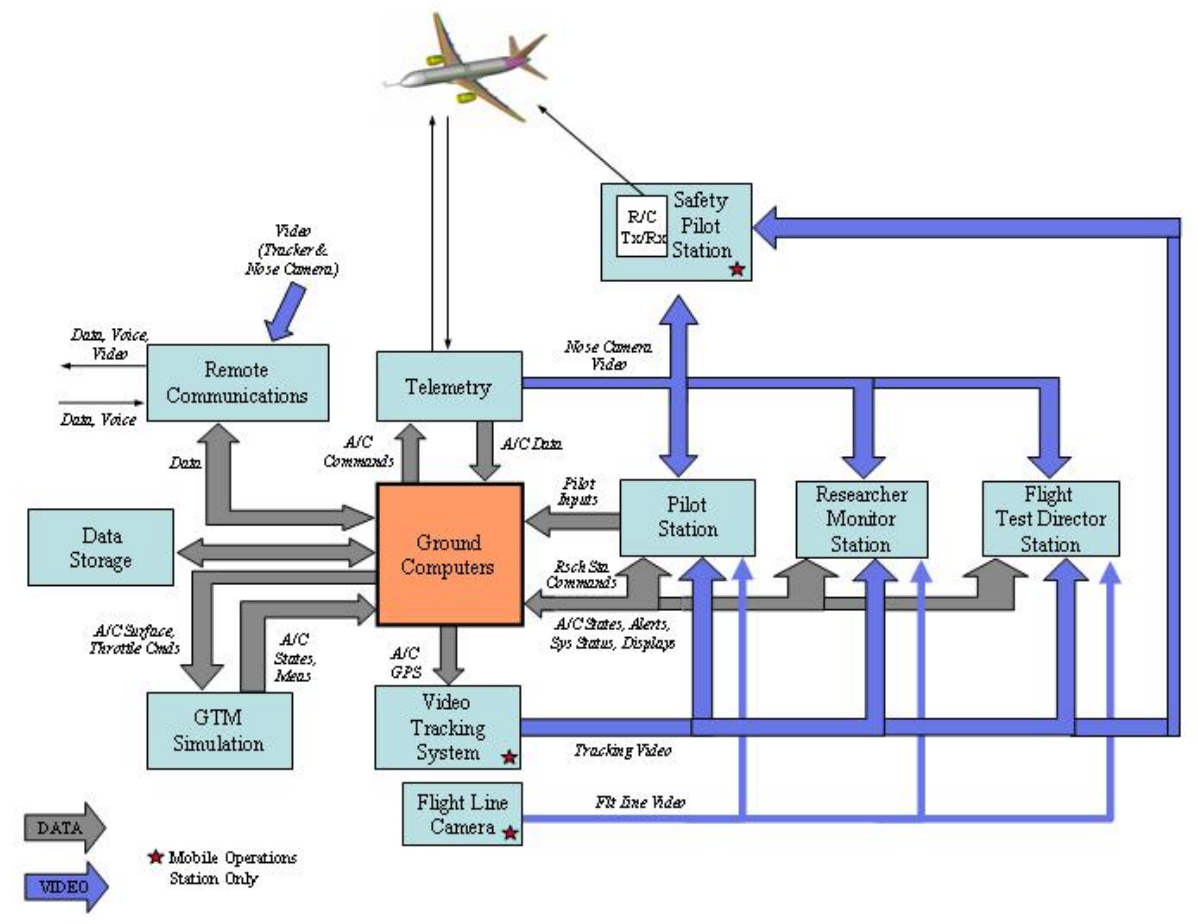

Figure 1. Block Diagram of Ground Facilities Functionality

The fundamental purpose of the GTM-GF is to provide the apparatus to accomplish the following:

- Permit research pilots to control the GTM;

- Receive, process, record and transmit GTM telemetry data;

- Display information to pilots, researchers, systems engineers and observers;

- Provide voice communications for experiment participants and observers;

- Provide data processing systems capable of computing control parameters on the ground to augment pilot control and/or remotely drive aircraft control surfaces;

- Provide real-time GTM simulation for training, testing and experiment reproduction.

The GTM Ground Facilities are designed to align with the overall development of the NASA Langley Research Center Systems and Airframe Failure Emulation, Testing and Integration (SAFETI) Research Laboratory [6]. The SAFETI Lab is being developed for validation testing of AvSSP technologies and to support long-term Aviation Safety and Security Program experimental research goals and objectives.

The GTM-GF will ultimately be the conjugation of two separate resources [7] - [8]: (1) the Mobile Operations Station (MOS) - a self-contained, motorized vehicle serving as a mobile research command/operations center, capable of deployment to remote sites when conducting GTM flight experiments [9]; and (2) the Base Research Station (BRS) - a laboratory based at the NASA Langley Research Center providing near-identical capabilities as the MOS. Figures 2 and 3 below outline the basic differences between hardware systems in the BRS and the MOS. 


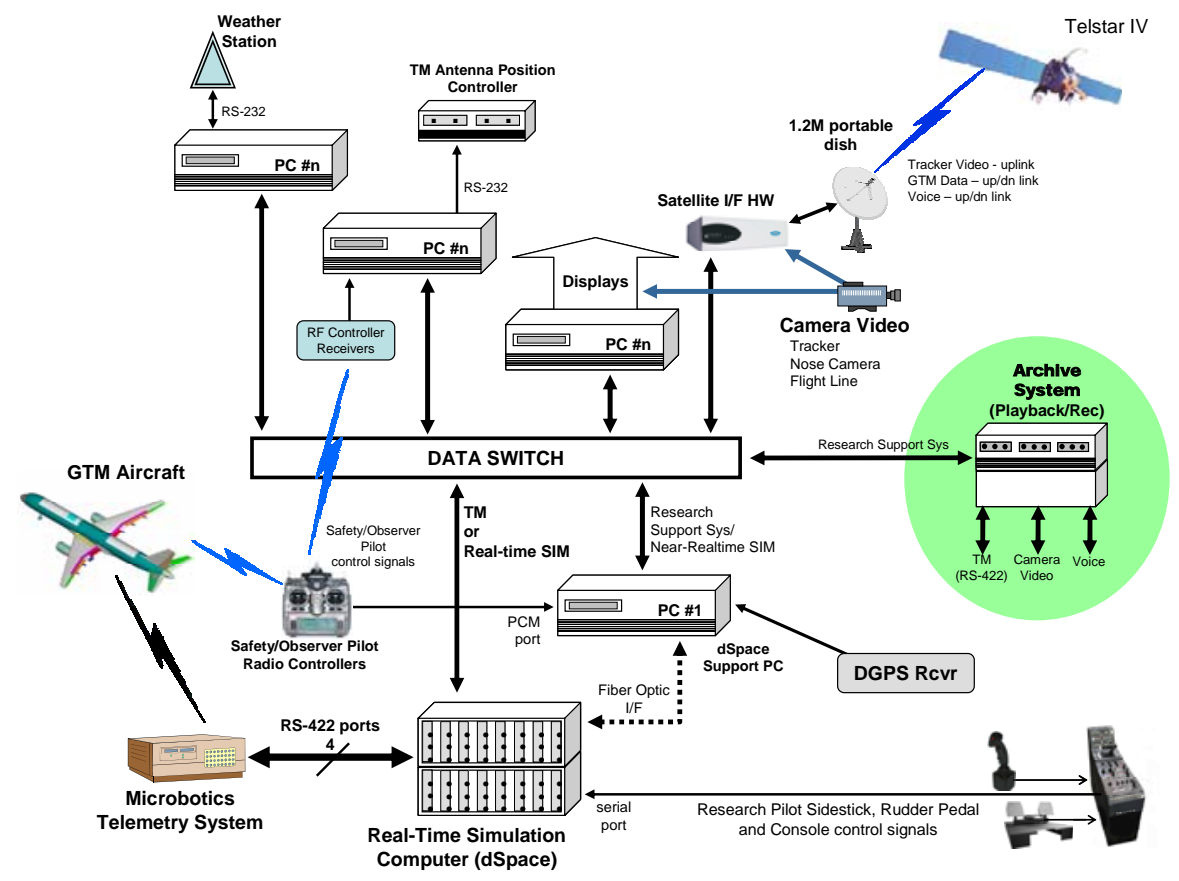

Figure 2. MOS Hardware Systems Overview

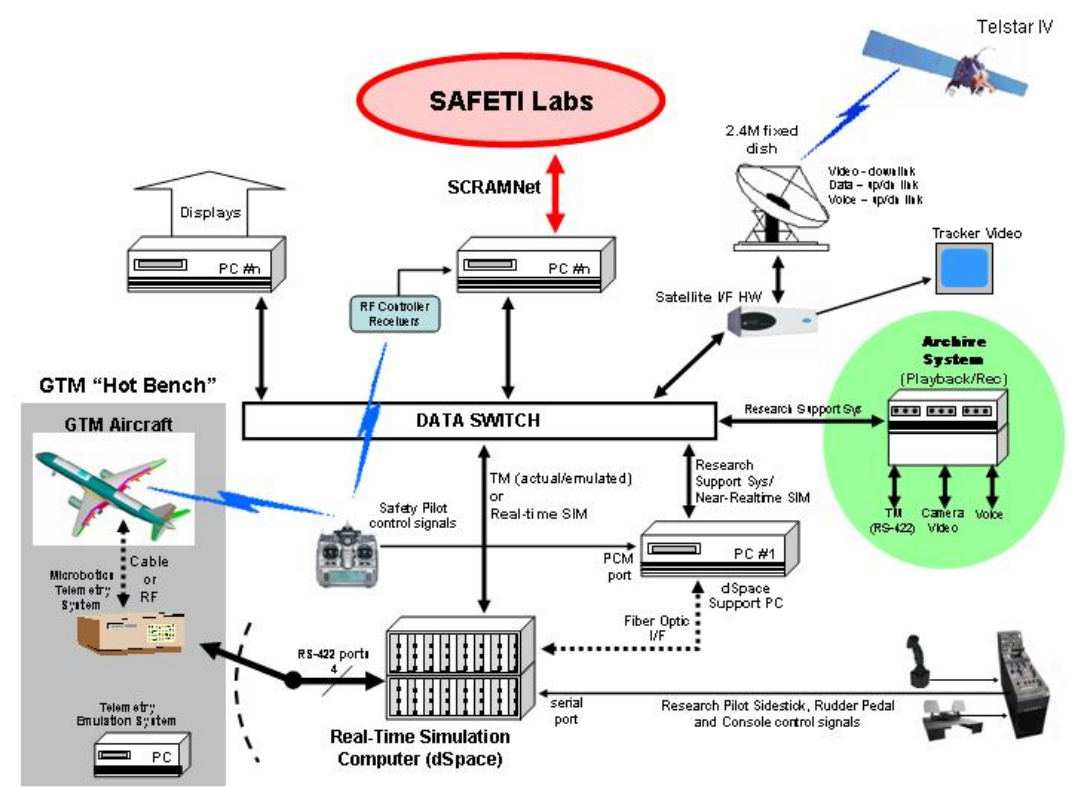

Figure 3. BRS Hardware Systems Overview

The overall GTM-GF architecture will also include the capability to link the BRS and MOS (data, video and voice) during GTM flight experiments. The BRS will be used for experimental software development and testing. The BRS also includes a "hot bench" work area which will accommodate hardware-in-the-loop test and evaluation with the GTM.

This paper provides a detailed description of the AirSTAR testbed ground facilities being developed at the NASA Langley Research Center. Section 2 describes the major hardware systems. Data archiving and playback is discussed in Section 3. Section 4 describes the Audio/Video Systems. The satellite-based data/video link is described in Section 5. A description of the GTM Video Tracking System is provided in Section 6. The Mobile Operations Station is described in Section 7. Section 8 discusses the Safety Pilot Station. Major software components are described in Section 9. A summary and some concluding remarks are provided in Section 10. 


\subsection{Major Hardware Subsystems}

Major hardware subsystems include the air-ground data link, computational resources, and user interfaces. Each of these subsystems is discussed in the following subsections.

\subsection{Air-Ground Datalink}

Communications between the AirSTAR aircraft and the GTM-GF are via a duplex serial data link using microwave RF transceivers, with a separate safety-pilot backup command RF link (ref section 8.0). The current experiment for which the GTM-GF is being configured will conduct flight maneuvers within a test range airspace volume of approximately 2 cubic statue miles ( 2 miles $\mathrm{x} 1$ mile $\mathrm{x} 1$ mile). To maintain effective telemetry communications coverage, the GTM-GF will employ a directionally-tracked, circularly-polarized antenna system. Analysis of the anticipated RF budget (involving transmitter powers, receiver noise floors, and antenna gains) supports the desired operation of the microwave links to slant ranges up to 2 statue miles with power margins in excess of 15 decibels. The AirSTAR telemetry system evolved from an air-to-ground datalink system developed by Microbotics Inc., and utilizes a proprietary encoding scheme based on the IRIG-106 standard. The telemetry system is comprised of a custom built airborne unit installed in the GTM and a custom built ground unit interfaced to the GTM-GF [10] - [12].

\section{Telemetry Airborne Unit (TAU)}

The TAU's primary function is to perform the multiplexing and de-multiplexing of the telemetry data for the GTM's on-board instrumentation and control systems. The on-board TAU is microprocessor-based, and incorporates hardware to assist in aircraft operations and instrumentation, including signal interface boards, safety switch, and power supplies. The TAU acquires data from various sensors aboard the GTM, including transducers indicating flight surfaces position, angle-of-attack probes, engine control units (ECU), MIDG II $^{\dagger \dagger}$, battery status, and employs a 'Flight Controller' module to multiplex this data for the air-to-ground telemetry stream. Conversely, the 'Flight Controller' module is used to de-multiplex the ground-to-air telemetry stream, distributing commands to GTM subsystems, including the flight surface servos. Additionally, video from the GTMs' nose camera will be transmitted via the RF link to the ground.

\section{Telemetry Ground Unit (TGU)}

The TGU's primary function is to perform the multiplexing and de-multiplexing of the telemetry data for GTM-GF client computers, and is housed in a standard 19" rack-mountable enclosure. The TGU interfaces to the GTM-GF computing system via four asynchronous serial (RS-422) ports that are used to receive flight data from the GTM and transmit control surface servo commands to the GTM. These ports, each running at 115200 baud, parse the telemetry data stream as shown in Tables 1 and 2.

\section{Table 1: Ports for Telemetry from GTM to GTM-GF (Downlink)}

\begin{tabular}{|c|c|c|c|}
\hline \multicolumn{4}{|c|}{ (Downlinked on S-band frequency $2240.5 \mathrm{MHz}$ ) } \\
\hline port 0 & \multirow{3}{*}{$\begin{array}{l}16 \text { Analog signals per port ( } 48 \\
\text { total) } \\
\text { includes surface positions, } \\
\text { angles of attack, air speed, } \\
\text { battery status, etc }\end{array}$} & \multirow{3}{*}{$\begin{array}{l}8 \text { digital inputs each port0 and } \\
\text { port1; } 16 \text { digital inputs port2 } \\
\text { (32 total) } \\
\text { includes system status codes, } \\
\text { gear up/down, etc. }\end{array}$} & \multirow{3}{*}{$\begin{array}{l}1 \text { serial stream each port0 and } \\
\text { port1 for engine control units } \\
\text { ( } 2 \text { total) } \\
\text { codes from left and right } \\
\text { ECU's }\end{array}$} \\
\hline port 1 & & & \\
\hline port 2 & & & \\
\hline port 3 & \multicolumn{3}{|c|}{1 serial stream from the MIDG II inertial package } \\
\hline
\end{tabular}

Additionally, the TGU receiver uses an RF sideband to receive video from the GTM nose camera, with this received video signal present at a rear-panel BNC connector.

\footnotetext{
${ }^{\dagger+}$ The MIDG II is a product from Microbotics Inc. which outputs GTM rotational rates, attitude, accelerations, GPS velocity and position, inertial navigation velocity and position (filtered velocity and position from GPS measurements and inertial measurements), magnetometer data, and GPS satellite data, all in a very small, lightweight package.
} 
Table 2: Ports for Telemetry from GTM-GF to GTM (Uplink)

\begin{tabular}{|c|c|}
\hline & (Uplinked on L-band frequency $1835.5 \mathrm{MHz}$ ) \\
\hline port 0 & \multirow{2}{*}{$\begin{array}{l}16 \text { Pulse Width Modulated (PWM) signals each ( } 32 \text { total) } \\
\text { servo commands }\end{array}$} \\
\hline port 1 & \\
\hline \multirow{2}{*}{ port 2} & 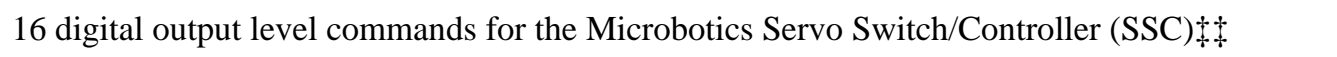 \\
\hline & This message is ignored at the airborne unit if SSC auxiliary channel defined as a digital input \\
\hline port 3 & RTCM DGPS corrections to GTM \\
\hline
\end{tabular}

The rate for GTM-to-Ground telemetry (excluding MIDG II) data is configurable within a range of 200 to $250 \mathrm{~Hz}$, and for AirSTAR operations is set to $216 \mathrm{~Hz}$ to accomplish the required data rates. The MIDG II provides INS data at $50 \mathrm{~Hz}$. Consequently, a 'subframe' period of approximately $4.6 \mathrm{~ms}$ is established for parameters downlinked on ports 0-2, while a 'major frame’ period of 20ms is established for MIDG II data downlinked on port 3.

Calculations indicate that downlink data from ports 0-2 (40 Bytes per port) will require $2.77 \mathrm{~ms}$ ( $60 \%$ of a minor frame) to arrive at GTM-GF computers. MIDG II INS data downlinked on port 3 (160 Bytes) are estimated to require approximately $11.11 \mathrm{~ms}$ ( 2.37 subframes $=55 \%$ of a major frame) to arrive at GTM-GF computers.

Calculations indicate that uplink data to ports $0-2$ ( $\leq 38$ Bytes per port) will require $2.64 \mathrm{~ms}$ ( $57 \%$ of a minor frame) to be received into the GTU. Thus, GTM-GF computing systems must process all parameters within an estimated $20 \mathrm{~ms}-(11.11 \mathrm{~ms}+2.64 \mathrm{~ms}) \cong 6.25 \mathrm{~ms}$.

\subsection{Computational Resources}

Scaled flight research has demanding data system frame timing deadlines that require the deterministic program execution rate provided by a real-time operating system (RTOS). The GTM-GF computing system is capable of accomplishing three primary tasks:

1. receiving telemetered GTM state data (e.g. sensor outputs, discretes, etc.), converting these data to calibrated engineering units in real-time, and transmitting control-surface and engine commands and other required state data to the GTM via the telemetry system;

2. processing, in real-time, researcher-provided algorithms, including

- flight control algorithms to compute control-surface and engine commands in response to pilot inputs and/or aircraft state data;

- failure detection and isolation (FDI) algorithms, upset recovery algorithms, and guidance algorithms;

3. providing real-time, hardware-in-the-loop (HIL) simulation of the GTM, including the capacity for integrating synthetic vision display components and terrain databases for flight control by the research pilot.

Non-time-critical computing tasks such as generation of user displays are managed by Intel®/Windows ${ }^{\circledR}$-based Personal Computers.

Time critical computing tasks will be managed by the dSPACE® computing platform. The dSPACE Real-Time Operating System (RTOS) was chosen to control the GTM while flying research control laws. dSPACE, which

\footnotetext{
\# The Microbotics Servo Switch/Controller (SSC) is a highly configurable, multiplexing switch for servo command signals. It allows the dynamic selection of several sources of pulse signals for servos. Pulse sources include asynchronous serial communication packets from a control computer, pulse signals from a conventional RC receiver or other servo pulse generator, and user definable constant signals.
} 
integrates target processor hardware, C code, analog and digital input/output, and control law block diagrams, was chosen because it was based on Matlab ${ }^{\circledR}$ and Simulink ${ }^{\circledR}$ products developed by The Mathworks, Inc. The dSPACE platform is a good fit with existing commercial aircraft simulations based on tools developed by The Mathworks, Inc. Matlabs' plotting and scripting features facilitate manipulation of wind tunnel data sets to obtain aerodynamic data parameters and verify control algorithms against check data. The graphical data flow characteristics of Simulink are a close fit to the characteristics of control law diagrams in aerospace documentation. The use of dSPACE reduces the amount of custom programming necessary to integrate the aircraft telemetry subsystems. The commonality between dSPACE and the simulation tools facilitates exercising the software at each stage of development with a realistic execution environment, and rapidly testing various HIL configurations.

\subsection{User Interfaces}

GTM-GF operations will be conducted by personnel located at three primary work areas: the Flight Research Station, the Operations Command Station, and Operations Engineering Station. Each of these work areas are described below.

\subsubsection{Flight Research Station (FRS)}

The GTM pilot and research projects' principal investigator (PI) will be located in the FRS. This station provides the means to fly the GTM manually or automatically by engagement of a researcher-provided autopilot flight control system implemented in the dSPACE computer. The FRS will provide all necessary pilot controls and a variety of displays for the pilot and PI. Figures 4 and 5 show photographs of the FRS and BRS hardware, respectively.

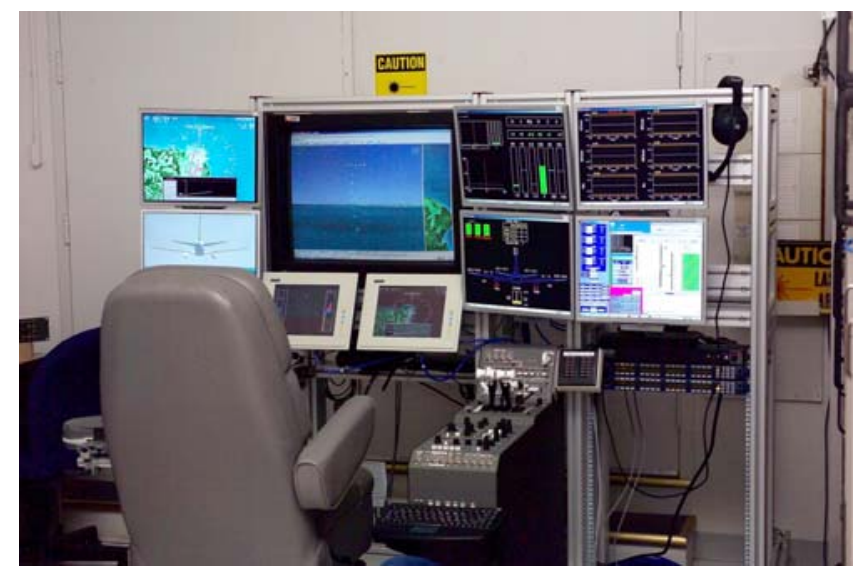

Figure 4. Flight Research Station in BRS

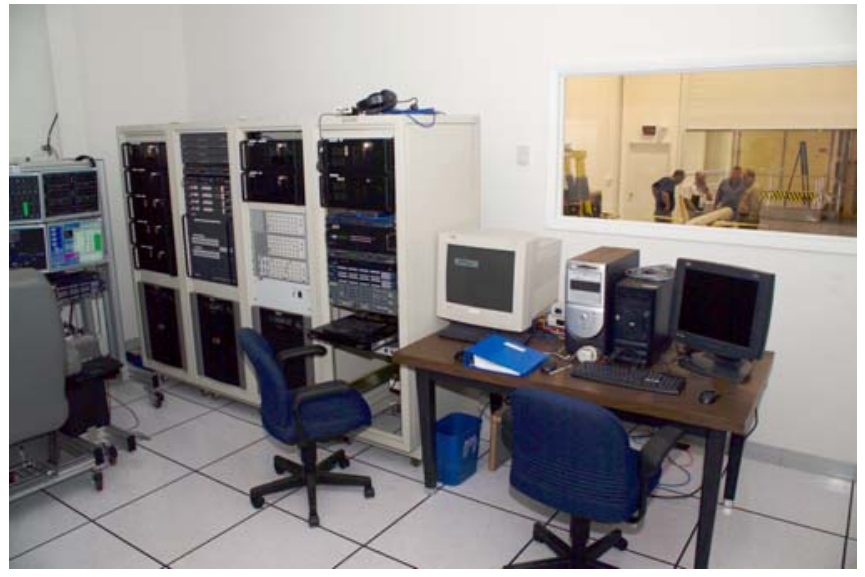

Figure 5. Photograph of BRS subsystem hardware - note view into "hotbench" area 
The display layout for the FRS is shown in Figure 6.

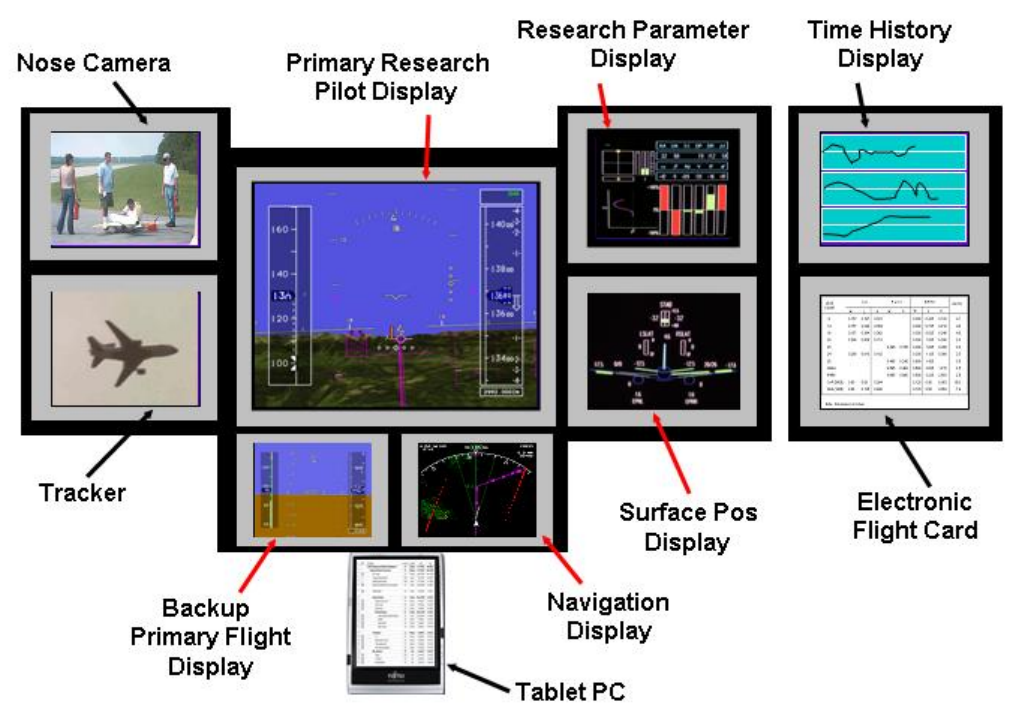

Figure 6. Display Layout for the Flight Research Station

Displays generated for the FRS will include the Primary Research Pilot Display, the Navigation Display, as well as other displays. These displays are described below.

\section{GTM Primary Research Pilot Display (PD)}

The PD will serve as the pilot's primary indicator of flight information, incorporating display elements typically presented as part of the Primary Flight Display (PFD) found on modern commercial and business aircraft. The PD provides GTM flight information, including but not limited to, attitude and heading, airspeed, altitude, track angle, flight path angle, load factor, angle-of-attack, and sideslip angle. The PD can also be configured to integrate synthetic terrain or nose camera video into its background, and provide programmable primary display symbology for the research pilot. Figure 7 shows an example of a potential PD display format with a synthetic vision background.

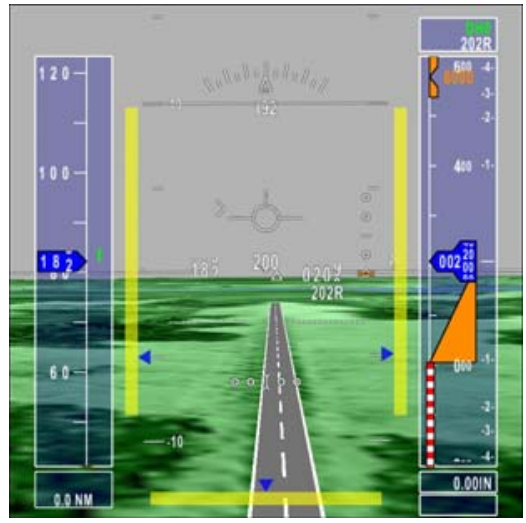

Figure 7. Prototype GTM Primary Research Pilot Display 


\section{GTM Navigation Display (ND)}

The ND will serve as the pilot's primary indicator of situational flight information, incorporating display elements typically presented as part of the Navigation Display found on modern commercial and business aircraft. The ND will serve as the pilot's primary indicator of the GTMs' location during flight tests, and can be configured to provide a variety of indicators and alerts, including a test range map with boundaries, runway depiction, vehicle track angle, desired horizontal path waypoints, and predicted path in a specified time period. Figure 8 shows an example of a potential ND format.

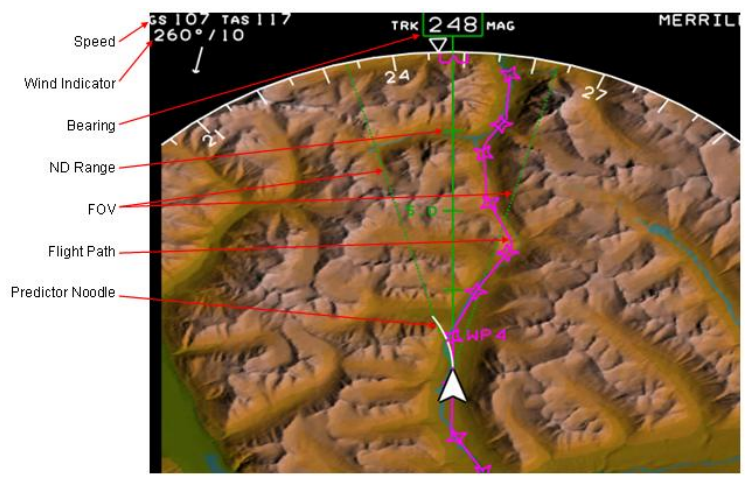

Figure 8. Prototype GTM Navigation Display for the Research Pilot Station

\section{Other Displays}

In addition to the Primary Research Pilot Display and Navigation Display, the GTM-GF provides for the generation and distribution of appropriate graphical displays for all flight test personnel including:

- $\quad$ any available GTM state data and/or experiment parameters

- $\quad$ camera video (e.g. tracking system, GTM nose camera, other on-board and ground-based cameras)

- $\quad$ any available health and/or status information associated with GTM and GTM-GF subsystems (e.g. onboard battery power, TM link signal strength, weather data, etc.)

\subsubsection{Operations Command Station (OCS)}

The Flight Test Director, who has the responsibility of coordinating all flight test related activities, and another researcher will be located in this area. The OCS will be provided with six display units, and the capability to configure and select among all available video sources as desired. The OCS will provide for monitoring and control of all audio communications between flight test participants. The OCS will also provide the capability to conduct researcher-specific activities such as:

- $\quad$ selecting test parameters for display;

- $\quad$ setting test parameters that control test conditions;

- $\quad$ setting simulated fault conditions;

- $\quad$ performing pre/post-flight analysis of recorded flight data, including running the GTM simulation.

Figures 9 and 10 show the general layout for the OCS and a photograph, respectively. 


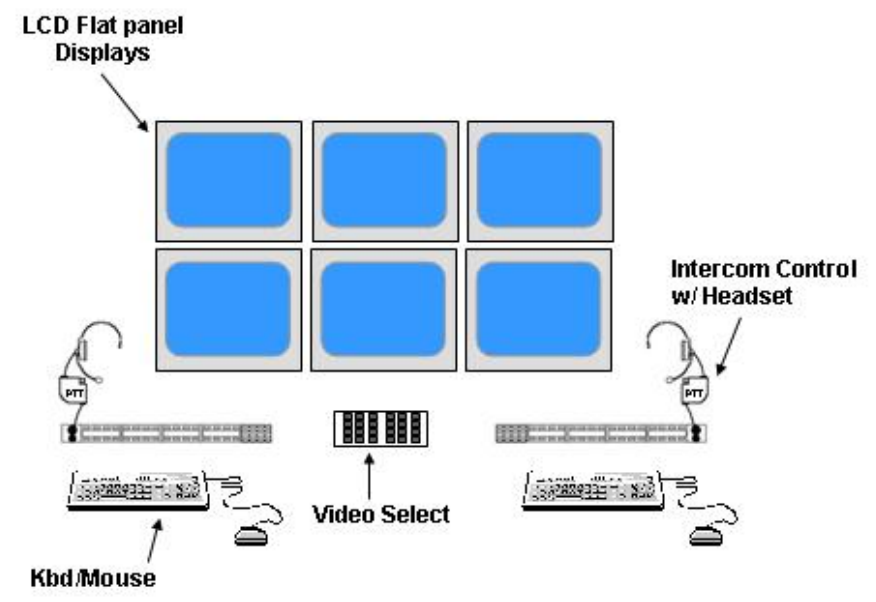

Figure 9. General Layout for Operations Command Station and Operations Engineering Stations

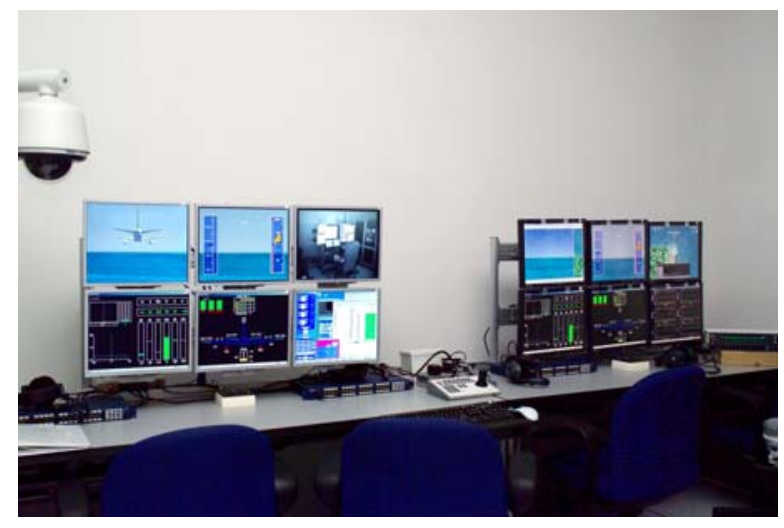

Figure 10. Photograph of Operations Command Station and Operations Engineering Stations in BRS

\subsubsection{Operations Enqineering Station (OES)}

Hardware and software support engineers will be located in this area. These support engineers will be responsible for monitoring and maintaining GTM-GF hardware and software subsystems during flight test operations. The OES will be similar in appearance to the OCS, and will have keyboard and mouse access to all GTM-GF computers, as well as the satellite terminal and data network (see Figures 9 and 10).

\subsection{Data Archiving and Playback}

Integral to the mission of AirSTAR scientific research is data collection and storage. The success of post-flight analysis hinges on the accurate and properly registered capture of GTM state parameters and GTM-GF process elements. With valid and complete data the stated goals of investigating new aerodynamic technologies can be more fully realized. The GTM-GF is equipped with an Asynchronous Real-Time Multiplexer and Output Reconstructor (ARMOR) subsystem. This system element consists of an Apogee Labs Model 4800 Digital Recorder Unit (DRU) interfaced to an Apogee Labs MITC FALCON Model 4303 Multiplexer /Demultiplexer. The multiplexing system merges multiple data sources, including GTM PCM telemetry data, $10 \mathrm{Mb} / \mathrm{s}$ Ethernet, voice, time code signals and video into a composite stream for digital recording. A plug-in Recorder Interface Module (RIM) provides a link to the DRU, which provides a recording interface to RAID 1 hard drives, with $90 \mathrm{Mb} / \mathrm{s}$ data transfer rate. 
This ARMOR system is configured to provide playback of all recorded GTM flight data parameters, physical camera video outputs, synthetic display video outputs, audio loops, and UTC time tag information, with partial or complete data reconstruction, including downlink/uplink telemetry to the GTM, camera video, audio loops, and master UTC information, allowing new real-time simulations to execute using recorded state data. Partial reconstruction provides the capability to perform engineering tests of the air-ground system, and debugging of anomalous behavior.

\subsection{Audio/Video Systems}

The GTM-GF Audio System is described in Section 4.1 and the Video System is described in Section 4.2.

\subsection{GTM-GF Audio System}

The GTM-GF provides the communications infrastructure to allow all key participants in GTM flight test activities to maintain voice contact. The heart of the intercom system is the Clear-Com Inc. MicroMatrix ${ }^{\circledR}$ 24channel digital intercom system. Onboard slots for interface cards permit seamless interfacing with telephones, twoway radios, party-line intercoms, and wireless belt packs. Set up of point-to-point communications, groups, monitoring lines, and "virtual" party lines for all flight test participants is accomplished using interactive and menudriven Windows-based software. The intercom master control system is operated by the flight test director during GTM flight test operations.

\subsection{GTM-GF Video System}

The GTM-GF provides the video hardware infrastructure to allow all video camera output signals and all computer generated displays to be amplified and split as necessary for distribution to key participants in GTM flight test activities. The heart of the GTM-GF video distribution system is Extron Electronics' CrossPoint Plus 24x24 matrix video switch, an ultra-wideband analog RGBHV matrix switcher, capable of distributing any of 24 video inputs to any combination of 24 video outputs simultaneously. Additionally, a Crestron Inc. MC2E controller is interfaced to the video matrix switch, permitting users to operate the matrix switch from the Flight Research Station, Operations Command Station or the Operations Engineering Station.

\subsection{Satellite-based Data/Video Link}

In order to provide the capability to monitor MOS GTM flight test activities at the Base Research Station located at Langley Research Center, a satellite backbone transmission system will be utilized to (1) transmit GTM telemetry state data, optical video and voice communication from MOS to BRS, and (2) transmit desired support data and voice communications from BRS to MOS. The major communications system elements comprising the satellite system consist of a fixed base station satellite dish, a deployable remote station satellite dish, and the necessary interface equipment. The Base and Remote Stations employ a satellite backbone transmission system augmented by data, video and voice communication facilities, and an Internet Protocol (IP) routing system. The IP routing system provides for multiple communication transmission types and automatic routing path requirements. The base station consists of a 2.4 - meter dish antenna and rack mounted satellite, video, audio, and routing equipment. The remote station consists of a 1.2-meter dish antenna contained in two field carrying cases, a field case for the antenna RF equipment, and satellite, video, audio, and routing equipment mounted in a separate field carrying case. The remote station is further augmented by a wireless video and audio transmission system, which provides for line of sight transmission of tracking video and voice communications from the Video Tracking Station.

The satellite system will rely on an Immeon network communications link. The Immeon System is a joint venture between Loral Skynet and ViaSat. The Immeon System is unique in that it can supply connectivity between satellite stations via a satellite, in this case Telstar IV, on an as needed basis. This lowers satellite usage costs. The connection service is available on a 24 hours-per-day, 7 days-a-week basis and is provided by a teleport and network control system located in Atlanta, GA. The system communications are based on Internet Protocol (IP) addressing; fully automating all routing of video, data, and voice communications within the system. This is accomplished by using commercial off-the-shelf network routing equipment. The routing equipment will utilize telephony-switching equipment to accommodate voice communications. The camera optical data will be processed by a video encoder at the MOS site for satellite transmission, and translated back to video at the BRS site for display purposes. 
The satellite system will utilize two carrier frequencies within the U.S. Ku band, between 14.0 and $14.5 \mathrm{GHz}$. As this frequency is in a non-federal government band, it was coordinated through the Federal Communications Commission (FCC) and is subject to FCC jurisdiction. Consequently, there is a one-year duration of use with renewal options.

The 2.4Meter fixed dish at the BRS will employ an 8Watt power amplifier to achieve a $1.0 \mathrm{Mbps}(1 \mathrm{Mbps}=$ 1,048,576 bits-per-second) uplink data rate. The 1.2Meter deployable dish at the MOS will employ a 25Watt power amplifier to achieve a 2.048Mbps uplink data rate.

As the Telstar IV system is comprised of satellites located 22,000 miles above the equator in geosynchronous Earth orbit, closed-loop operations involving GTM control inputs generated at the BRS will incur a 0.5sec (minimum) latency. For this reason, remote piloting of the GTM from the BRS is not currently planned.

\subsection{Video Tracking System}

In order to provide the capability to monitor the GTM in flight, a ground-based video tracking system will be deployed with the MOS and will track the airborne vehicle using cameras and electronic equipment. The tracking video could be used by safety pilots to help fly the GTM to the MOS if a takeover is required when the GTM is beyond the line-of-sight. This video tracking system will be augmented by a wireless video and audio transmission system, to provide for transmission of tracking video and voice communications from the video tracking system to the MOS. The trackers' limited maximum slew rate (6 degrees per second estimated) dictates that the tracker be setup at a location offset one to two statute miles from the MOS, and within line-of-sight of the MOS wireless video receiver. This location will be called the Tracking System Station (TSS), and an operator will be required to operate the system. The TSS will employ a two-way communication link to the intercom master control system located at the Operations Command Station. The tracking system is capable of receiving GTM GPS/DGPS position data in order to augment the system's ability to maintain GTM tracking lock, and tracking video will be transmitted to the MOS for display as desired by users at the various operations stations.

The GTM Video Tracking System is a portable and fully self-contained system, designed and constructed by Celestial Computing Corp. The tracker can be disassembled and transported in six travel cases, each case having a maximum allowable weight of 120 pounds. The tracking system is comprised of a portable pier tripod assembly, supporting optical acquisition lenses and video cameras mounted on a motorized 2-axis gimballed system. The system includes an Automated Telescope Startup Unit (ATSU) with precision home and park positions to .001 degrees, with electronic Periodic Error Correction (PEC) and Charge Coupled Device CCD port for astronomical self-guiding, and is capable of computerized full-sky "Go-To" pointing, which can be set up for remote or local operation. The tracker is capable of equatorial and alt-azimuth alignment and tracking, with adjustable slew speeds up to 6.0 degrees per second. This hardware is interfaced to a PC running customized software, which sends commands to the gimbal system to maintain tracker lock on the GTM.

\subsection{Mobile Operations Station (MOS)}

The Mobile Operation Station is a deployable remote laboratory site that will be employed as the central research and command center for GTM research flight operations. In addition to all of the hardware and software components utilized in the BRS, the MOS contains the ancillary support subsystems required for operation in the field, including, power generator, Un-interruptible Power Supply (UPS) back-up subsystem, restroom facility, kitchenette, small meeting/work area, walk-on roof with safety rails, leveling jacks, and a self-stowing, deployable side awning. In addition to all interfaces available in the BRS, the MOS provides external power and data interfaces for additional subsystems, including, Safety Pilot Station, TSS video modem and Flight Line video camera, wireless transmitter for GPS data, VHF and cellular communications devices, LAN/internet, 1.2 meter portable satellite dish, and 120VAC "Shore" power feeds.

The GTM MOS is built on a Freightliner ${ }^{\circledR}$ chassis and has the following specifications:

- $\quad$ GVWR - up to 54,000 lbs.;

- $\quad$ Length - 40 feet;

- $\quad$ Rear Axle - Tandem, up to 40,000 lbs.;

- Wheelbase - 290";

- Engine: CAT C7, $330 \mathrm{Hp}$. 
The vehicle's mission goal of providing a self-contained mobile research laboratory environment defines the design and minimum performance standard requirements of the Aft Body area. The Aft Body area is a self-contained/selfsupporting laboratory consisting of four (4) sectioned off areas that will provide support to the three (3) users operations stations, four (4) full size equipment racks, two (2) half size equipment racks, and a Galley with a kitchen and lavatory area. Figure 11 shows the MOS floor plan.

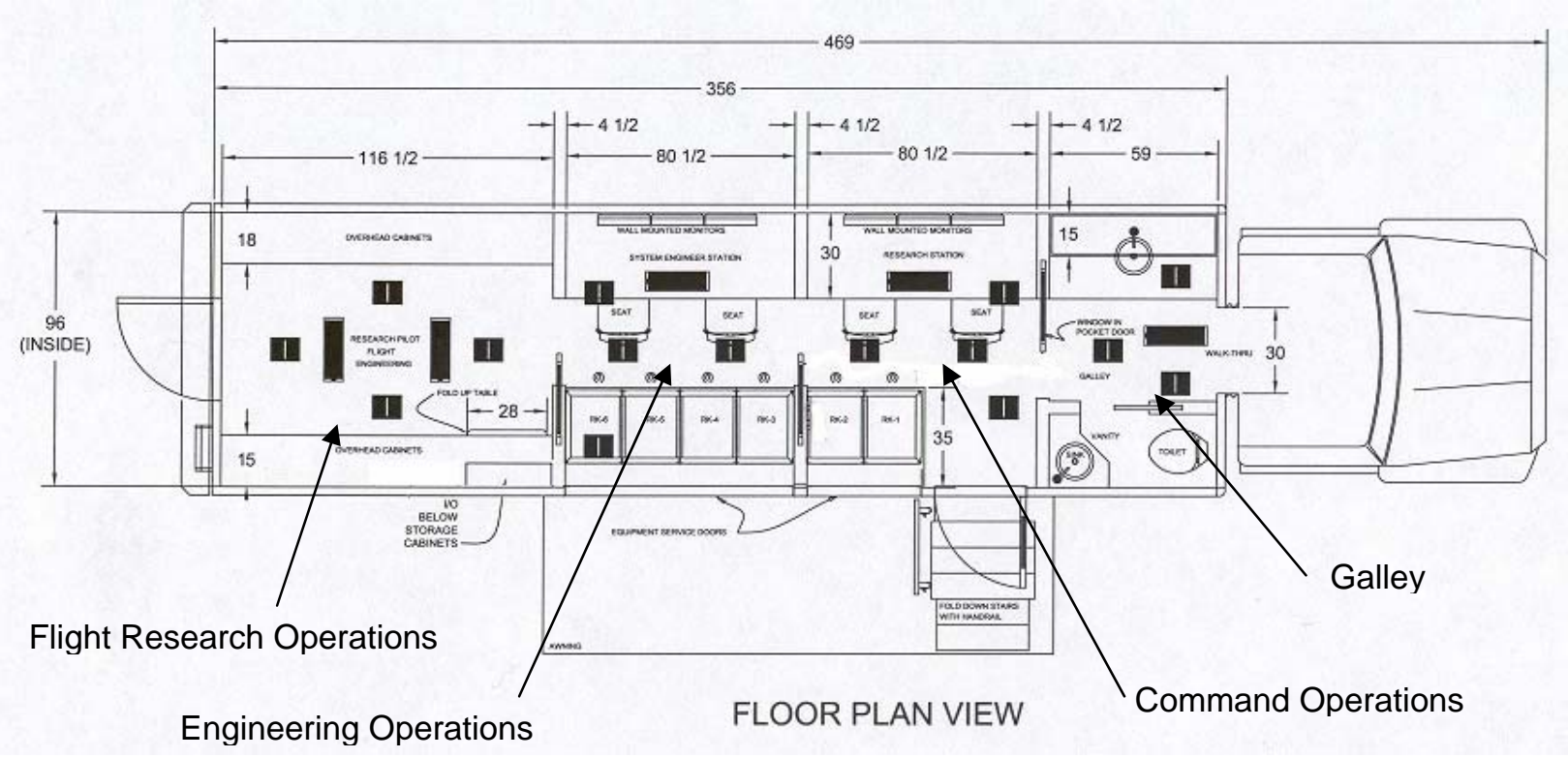

Figure 11. Mobile Operations Station Floor Plan View

\subsection{Safety Pilot Station}

In recognition of the fact that research flight will be increasingly dynamic in nature and will seek to set up highrisk/off-nominal (e.g. large angle-of-attack and sideslip) flight conditions it is deemed necessary to retain the role and inputs of a conventional $\mathrm{R} / \mathrm{C}$ pilot towards enhancing overall mission safety. This capability to host a secondary controller, referred to as the safety pilot, has the following attributes.

- The safety pilot is located externally to the MOS vehicle to provide a line-of-sight view of the GTM so as to afford direct observation of craft attitude when possible. If takeover is required when the GTM is beyond the line-of-sight, the safety pilot will use the nose camera video, tracker camera video, or a combination of the videos to fly the GTM back into line-of-sight.

- The safety pilot inputs originate from a conventional handheld $\mathrm{R} / \mathrm{C}$ transmitter that is configured to continuously emanate a special safety channel signal.

The avionics of the GTM aircraft are engineered to receive the safety channel signal for the purpose of discriminating among four flight control cases:

$>$ ground research pilot inputs for advanced/general experiment conditions;

$>$ ground safety pilot inputs for basic/aborted experiment conditions;

$>$ onboard return-to-home autopilot inputs;

$>$ onboard range-safety inputs for abnormal/terminated flight conditions.

- The safety pilot has a dedicated two-way audio communication channel with the Flight Test Director Station and the Research Pilot Station.

Figure 12 illustrates how the Safety Pilot Station interfaces to the MOS. 


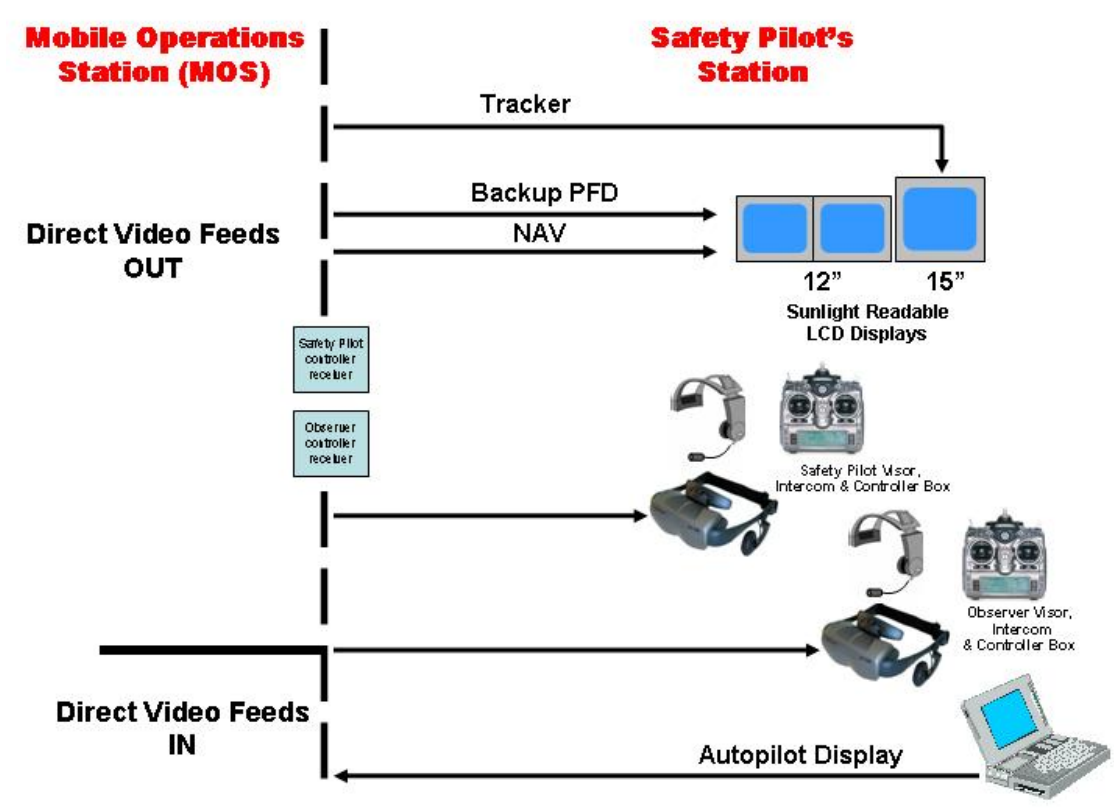

Figure 12. Safety Pilot Station Interfaces

\subsection{Major Software Components}

The GTM-GF system network architecture is shown in Figure 13.

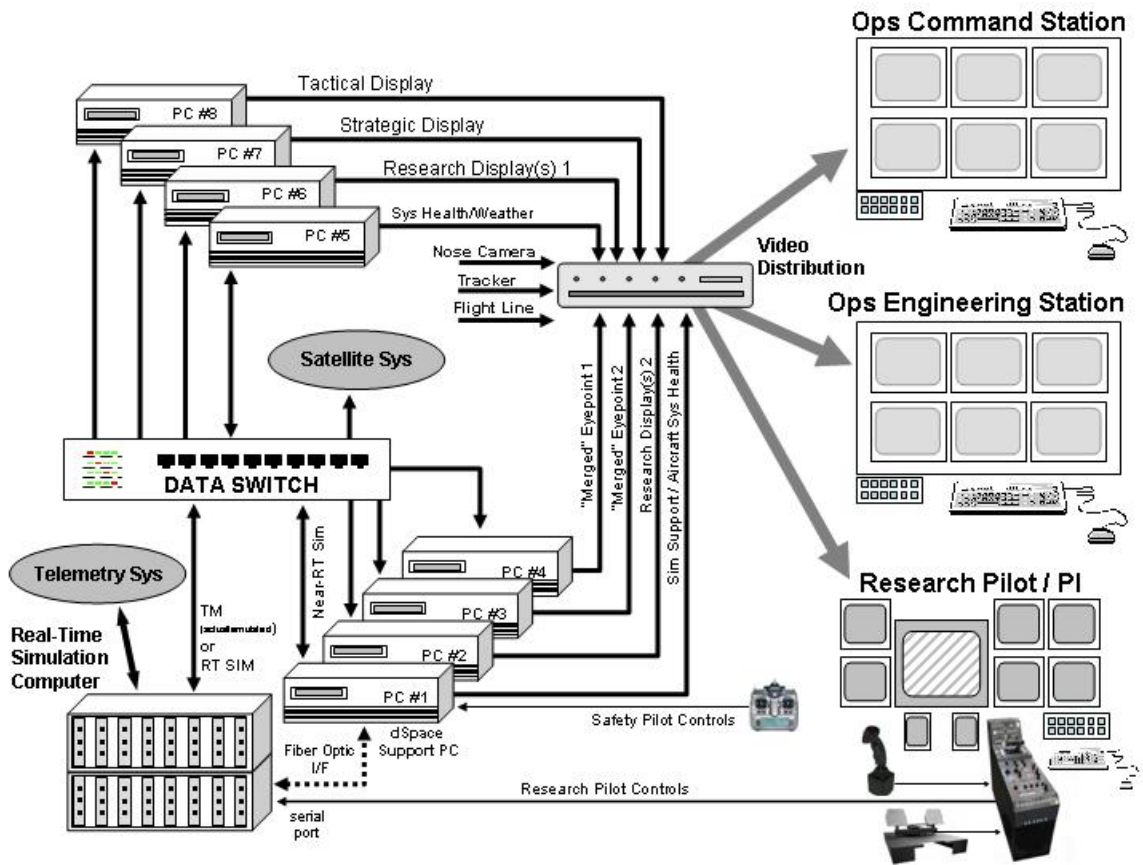

Figure 13. GTM Ground Facilities System Network Architecture

The major software components will be contained within eight PCs and a Real-Time Simulation Computer (dSPACE), shown in Figure 13. There are three broad components of the overall GTM-GF software architecture, the simulation, the displays, and the networking component for both the BRS and the MOS. These are discussed in Sections 9.1 and 9.2 . 


\subsection{Simulations}

The GTM simulation is capable of being executed in both the BRS and the MOS, and will acquire pilot and researcher input signals and generate six Degree of Freedom (6DOF) flight dynamics and sensor data, and emulate the GTM down-link telemetry stream. The simulation will run on a Personal Computer (PC) platform with the Windows operating system, using the Matlab/Simulink programming tools. The GTM simulation is referred to, in this document, as the Flight Dynamics Model (FDM), and will operate in three distinct configurations:

- Personal Computer (FDM-PC), standalone, near real-time operation, on one or more PCs;

- Piloted Simulation (FDM-PSIM), real-time simulated operation on the dSPACE platform;

- Hardware In the Loop (FDM-HIL) real-time operation simulation on the dSPACE platform.

Each of these configurations is discussed below.

\section{FDM-PC}

The FDM-PC is a standalone Matlab/Simulink simulation operating in near real-time on a Windows -based PC platform. The program is configured to receive input from a selection of flight controller hardware, send flight parameter information to a selection of graphical visual display programs, and send emulated telemetry sensor and flight information to an Ethernet port in User Datagram Protocol (UDP) packets to support development and testing of GTM Ground Facilities software. This setup is shown below in Figure 14.

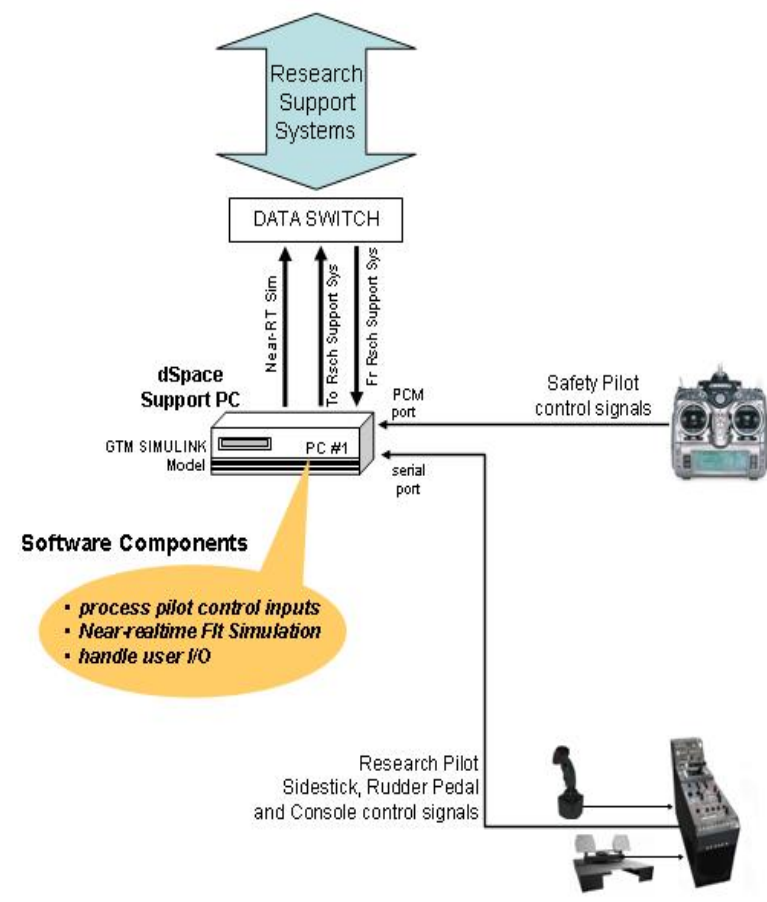

Figure 14. FDM-PC Configuration

\section{FDM-PSIM}

The FDM-PSIM operates on the dSPACE system without I/O to the GTM. This setup will receive controller input signals from the Research Pilot Station interfaced to the dSPACE system. Simulated telemetry data will be output as a UDP format and distributed through the Ethernet bus for display generation and archiving. This setup is shown below in Figure 15. 


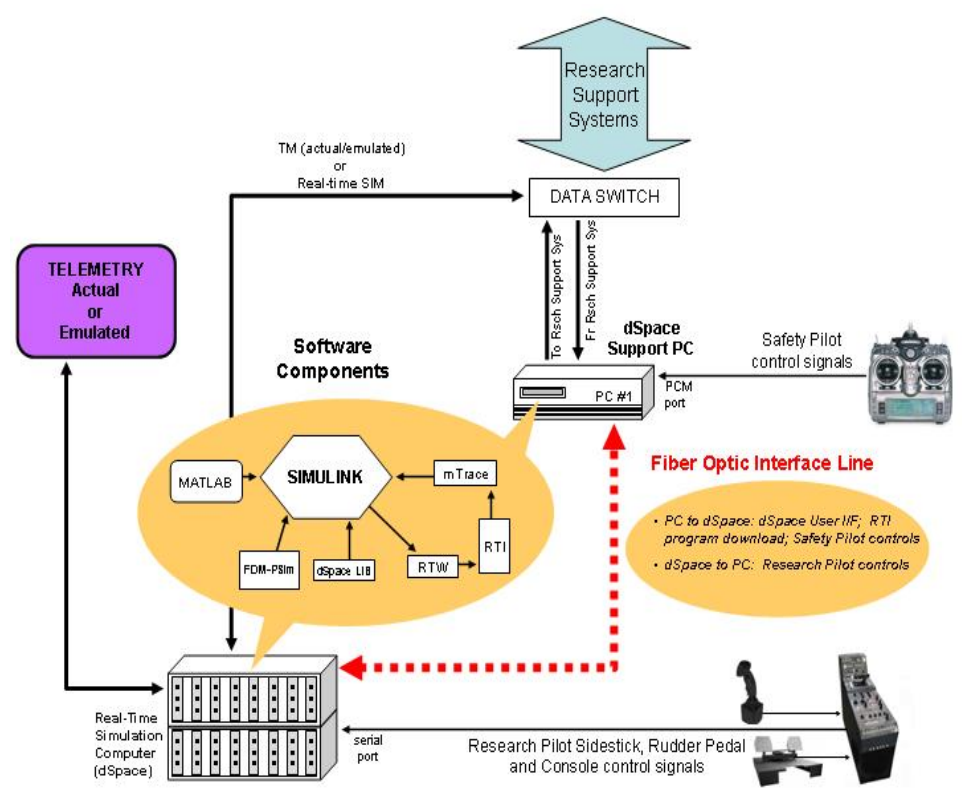

Figure 15. FDM-PSIM Configuration

\section{FDM-HIL}

The FDM-HIL operates with the GTM through the dSPACE platform. This setup receives controller input signals from the Research Pilot Station through dSPACE input ports. It will receive telemetry signals from GTM sensors through dSPACE RS-422 serial input ports, and outputs command signals to the GTM telemetry system through dSPACE RS-422 serial output ports. Additionally, all telemetry data (uplink and downlink), and aircraft surface position commands from the pilot controls will be output over Ethernet through the support PC for archiving and display generation. The simulation will have an option for archive playback of the GTM states and controls using information obtained from the GTM as a means for testing the FDM simulation fidelity. This setup is shown below in Figure 16.

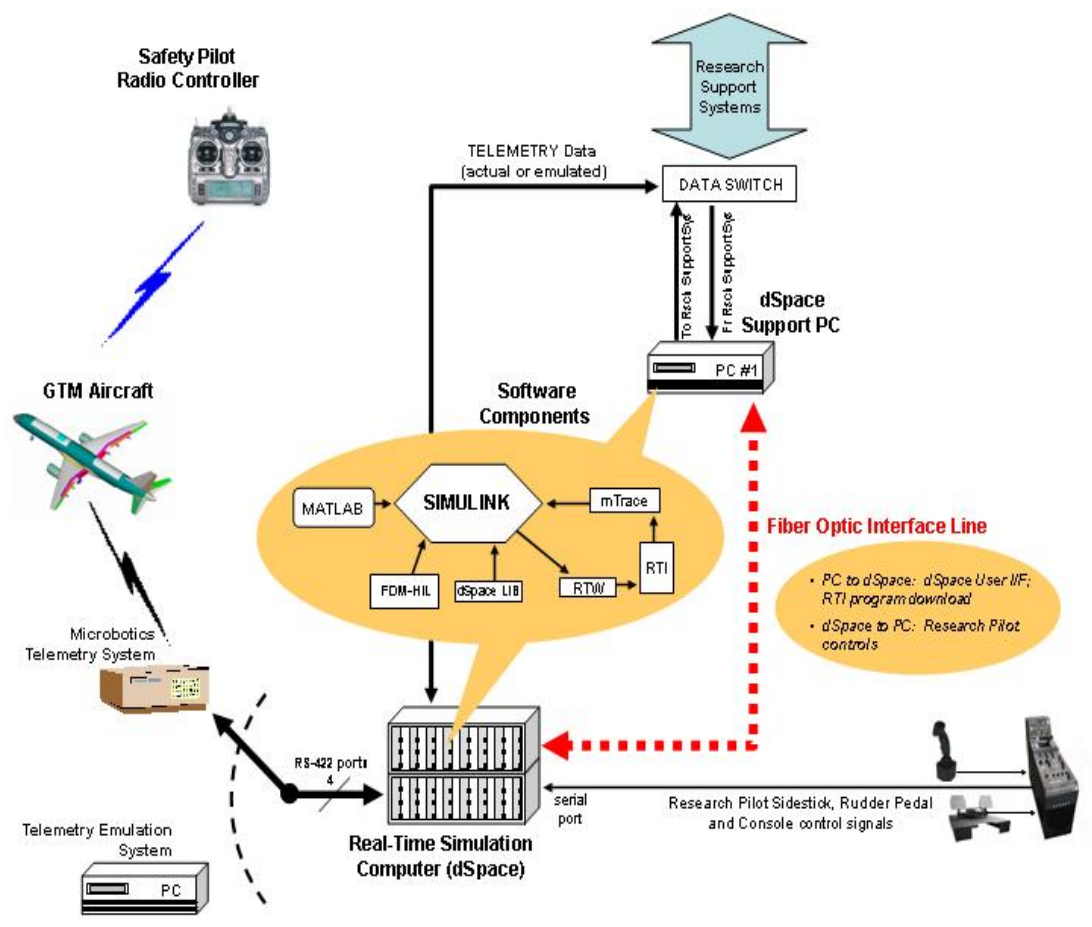

Figure 16. FDM-HIL Simulation Configuration 


\subsection{Networking and Displays}

Networking is an Ethernet application for distributing telemetry and control data from the control computer to all other computers and devices involved in the ground facilities (ref. figure 13). It is a self-contained application, but is used within the dSPACE-based Simulink applications to accomplish data output to the other systems of the ground facilities.

The displays will be generated by several independent software applications, which render synthetic terrain, GTM images, and informational graphics. These include synthetic vision underlays, primary and navigation display symbology, and standalone status displays. The networking applications used for displays are derived from work done for NASA's Synthetic Vision research project. The Synthetic Vision-based applications developed for the GTM-GF are comprised of three major components: the Message Manager, the Data Server and the Display Manager. Each of these components is described briefly below.

- Message Manager - the Message Manager receives the data streams from the GTM telemetry system, repackages this information, and sends it to the Data Server. This repackaging process is necessary since the amount of data and update rate of the data from the model can vary; however, the Data Server requires complete messages at a pre-defined rate.

- Data Server - the Data Server receives data from the Message Manager, performs any necessary postprocessing on the data, and places the data into the appropriate computers' memory. Post-processing of the data may consist of unit conversion (i.e., convert the data from micro-volts measured by the GTM's sensors to units that are more appropriate for creating and updating the displays). The Data Server also has the ability to record the data that it receives.

- Display Manager - the Display Manager reads the computers' memory to retrieve all of the data that is required to produce the desired displays. These displays include the Primary Flight Display (PFD), backup PFD, Navigation Display (ND), and all of the research displays.

The Message Manager is installed only on the computer that links with the GTM telemetry downlink data. The Data Server and Display Manager, however, are installed on all of the computers that generate displays.

\subsection{Concluding Remarks}

The GTM-GF is currently being developed and configured to support the experiment "Aerodynamic Model Validation For Upset Conditions". This experiment is being conducted to validate aerodynamic modeling methods and data for upset conditions which include conditions beyond the normal flight envelope. These conditions include stalls and post-stall departures from controlled open-loop pilot flight at high angles of attack and sideslip with high angular rates. The GTM-GF is scheduled to support flight testing activities in Spring 2006. Subsequently, the GTM-GF will be configured for testing automatic closed-loop control research systems that are being developed to prevent vehicle upset as a result of failures, or recover the vehicle from abnormal flight conditions.

\section{References}

1. Belcastro, Christine M.; Belcastro, Celeste M.; "Application of Failure Detection, Identification, and Accommodation Methods for Improved Aircraft Safety”, Proceedings of the American Control Conference, June 2001

2. Belcastro, Christine M.; Belcastro, Celeste M.; "On the Validation of Safety Critical Aircraft Systems, Part I: An Overview of Analytical \& Simulation Methods”, Proceedings of the Guidance Navigation and Control Conference, 2003

3. Belcastro, Celeste M.; Belcastro, Christine M.; "On the Validation of Safety Critical Aircraft Systems, Part II: An Overview of Experimental Methods", Proceedings of the Guidance Navigation and Control Conference, 2003

4. Jordan, T. L., Langford, W. M., Belcastro, Christine M., Foster, J. M., Shah, G. H., Howland, G., Kidd, R., "Development of a Dynamically Scaled Generic Transport Model Testbed for Flight Research Experiments", AUVSI Unmanned Systems North America 2004, AUVSI, Arlington, VA, 2004

5. Jordan, Thomas; Langford, William; Hill, Jeffrey S; “Airborne Subscale Transport Aircraft Research Testbed Aircraft Model Development”, Proceedings of the Guidance Navigation and Control Conference, 2005 
6. Belcastro, Celeste M.;" Overview of the Systems and Airframe Failure Emulation Testing \& Integration (SAFETI) Laboratory at the NASA Langley Research Center", Proceedings of the International Conference on Lightning and Static Electricity, 2001

7. Bailey, Roger M., Hueschen, Richard M.; "Functional Requirements for Development of Ground Facilities Supporting the Generic Transport Model”, Rev. 1.1, NASA Internal Report, April 30, 2004

8. Hostetler, Robert; “Generic Transport Model Ground Facilities Software Requirements Specification (SRS) ” v. 2.0; NASA Internal Report, 29 July 2005

9. Barnes, Kevin N.; "AirSTAR MOBILE OPERATION STATION PROCUREMENT REQUIREMENTS DOCUMENT”, rev 1.0, NASA Internal Report, 05April 2005

10. “GTM Communication Link Messages”, Microbotics, Inc., July 27, 2005

11. "PCM System, NASA LaRC GTM Custom Build”, Microbotics, Inc., April 27, 2004

12. “MIDG II Message Specification”, Microbotics, Inc., May 26, 2005 